\title{
Jefferson
}

\section{New phenotype of Parsonage-Turner Syndrome involving Radial and Proximal Median Nerve: A Case Report}

\section{Matthew McAuliffe, MD"; Nancy Vuong, MD1; Adam L. Schreiber, DO1,2}

'Department of Rehabilitation Medicine, Thomas Jefferson University Hospital, Philadelphia, PA

2Department of Rehabilitation Medicine' Jefferson Medical College, Thomas Jefferson University, Philadelphia, PA

\section{CASE REPORT}

HISTORY

A 74-year-old woman developed sudden severe left shoulder, arm, and forearm pain that suddenly developed. This pain lasted approximately 2 months. Approximately 1-2 months after the resolution of the first pain she developed a different type of pain which she described as a tingling, burning, pins and needle sensation that radiated from her shoulder past her elbow into the dorsal and palmar aspect of her hand in the thumb, index, and long fingers. Additionally, she developed numbness in her hand along the palmar thumb, index, and long fingers. Two months following the onset of the initial type of pain, she developed left hand weakness that progressively worsened.

\section{PHYSICAL EXAMINATION}

Decreased light touch over the posterior forearm, lateral forearm, absent light touch and pin over the dorsal radial aspect of the thumb, index and long fingers as well as the palmar aspect of thumb, index and longer fingers.

- Absent pronator teres and triceps reflexes.

- Manual muscle testing was $5 / 5$ in strength except as follows in the left upper limb:

shoulder external rotators, ellow flexors and flexor digitiorum profundus $4 / 5$ in the index and long fingers
- elbow extensors 315

extensor carpi radialis, extensory digitorum communis, flexor carpi radialis, flexor pollicus longus, flexor digitorum superficialis, and abductor pollicus brevis $0 / 5$.

\section{DIAGNOSIS AND TREATMENT}

Previous work-up included:

- Inconclusive EMG/NCS performed by a physical therapist,

- MRI of cervical spine which revealed non-focal degenerative changes

MRI of her left shoulder which revealed moderated tendinosis of the rotator cuff with focal tearing of the distal infraspinatus, mild subacromial bursitis and mild glenohumeral joint osteoarthritis. - MRI of her left brachial plexus did not show evidence of nerve injury. Patient was referred again for a repeat EMG/NCS.

\section{RESULTS}

EDX study, 4 months from onset of symptoms, revealed absent median and radial motor-sensory nerve conduction. EMG revealed complete denervation of FDP in the index and long fingers, as well as the pronator teres, partial reinnervation of the triceps, and no abnormal cervical paraspinal spontaneous activity. See Detailed results in Figure 1



Fig. 1 - Detailed Electrodiagnostic Results

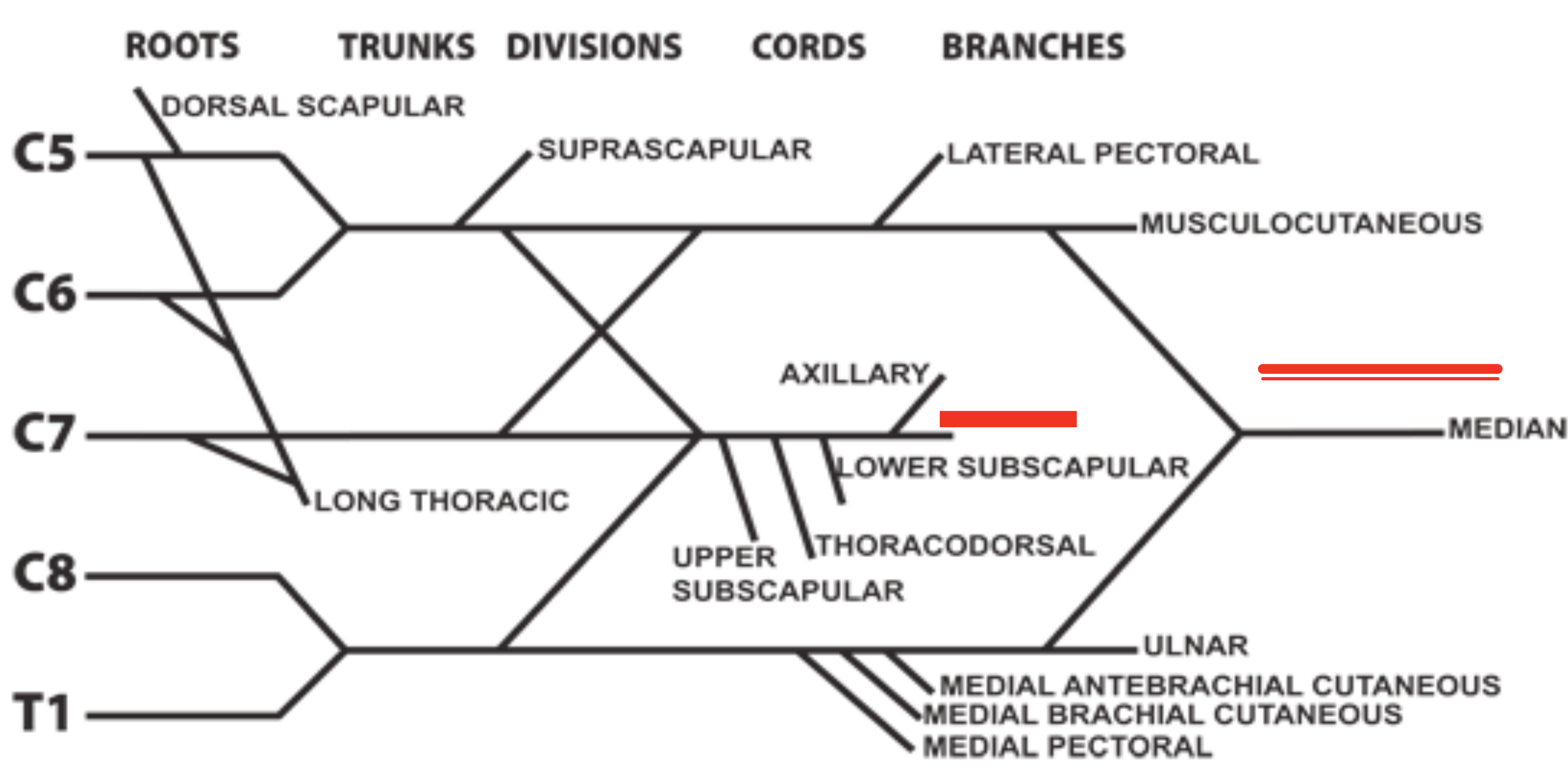

Fig. 2 - Brachial plexus demonstrating lesions at the radial and median nerve. Image used with permission from Gerald $J$. Herbison from http://jeffline.tju.edu/Education/programs/NEL/

\section{DISCUSSION}

This case fits the clinical description of neuralgic amyotrophy of Parsonage and Turner ${ }^{1,2}$. Typically, patients will have sudden severe neuropathic pain that resolves preceding the onset of weakness. ${ }^{3}$ Most patients will develop atrophy within the first 5 weeks of onset of symptoms. ${ }^{3}$ Alternative diagnosis in this case are less likely for the following reason. It is unlikely to be a concomitant radial neuropathy at the spiral groove and proximal median neuropathy with simultaneous onset. There is no evidence of myelinopathy (ie, demyelinating disease) as there is no conduction block to explain her weakness. Finally, exam and imaging were not consistent with more commo neuromusculoskeletal pathologies.

The etiology of neuralgic amyotrophy of Parsonage and Turner remains unclear, but it has been associated with infection, trauma, surgery, strenuous exercise, and vaccinations..$^{1,3}$ Often the symptoms begin at night, and are usually asymmetric. ${ }^{1,3}$

The diagnosis is often made by history and physical exam. EMG/NCS is useful in confirming the diagnosis and to localize the lesion or lesions. ${ }^{4}$ In some cases, EMG/NCS can also provide information on prognosis and recovery, since many patients will not recover fully from this condition. A large case series described long thoracic, suprascapular, upper trunk of brachial plexus and anterior interosseous nerve involvement attributed to autoimmune phenomenon. ${ }^{3}$ To our knowledge there are no reports of neuralgic amyotrophy of Parsonage and Turner to be presenting with concomitant proximal radial and median nerve involvement.

\section{CONCLUSION}

This case demonstrates an unreported phenotype of neuralgic amyotrophy of Parsonage and Turner involving both motor and sensory nerve fibres of the proximal radial and median nerves. This condition is best diagnosed by a careful history and physical exam. Clinicians should have a high index of suspicion for neuralgic amyotrophy of Parsonage and Turner in presentation of upper limb weakness, dysethesias, and parethesias that was preceeded by severe pain

\section{REFERENCES}

Parsonage MJ,

Turner JWA, Parsonage MJ. Neuralgic amyotrophy (paralytic brachial neuritis); with special

Van Alfen N, van Engelen BGM. The clincial spectrum of neuralgic amyotrophy in 246 cases

Schreiber AL, Abramove R, Fried GW, Herbison GJ. Expanding the differential of should pain: Parsonage-Turner syndrome. Journal of the American Osteopathic Association. 